\title{
Effects of coating and diametric load on fiber Bragg gratings as cryogenic temperature sensors
}

\author{
Meng-Chou Wu*, Ruth H. Pater, and Stanton L. DeHaven \\ NASA Langley Research Center, MS 231, Hampton, Virginia, USA, 23681-2199
}

\begin{abstract}
Cryogenic temperature sensing was demonstrated using pressurized fiber Bragg gratings (PFBGs) with polymer coating of various thicknesses. The PFBG was obtained by applying a small diametric load to a regular fiber Bragg grating (FBG). The Bragg wavelengths of FBGs and PFBG were measured at temperatures from $295 \mathrm{~K}$ to $4.2 \mathrm{~K}$. The temperature sensitivities of the FBGs were increased by the polymer coating. A physical model was developed to relate the Bragg wavelength shifts to the thermal expansion coefficients, Young's moduli, and thicknesses of the coating polymers. When a diametric load of no more than $15 \mathrm{~N}$ was applied to a FBG, a pressure-induced transition occurred at $200 \mathrm{~K}$ during the cooling cycle. The pressure induced transition yielded PFBG temperature sensitivities three times greater than conventional FBGs for temperatures ranging from 80 to $200 \mathrm{~K}$, and ten times greater than conventional fibers for temperatures below $80 \mathrm{~K}$. PFBGs were found to produce an increased Bragg wavelength shift of $2.2 \mathrm{~nm}$ compared to conventional FBGs over the temperature range of 4.2 to $300 \mathrm{~K}$. This effect was independent of coating thickness and attributed to the change of the fiber thermo-optic coefficient.
\end{abstract}

Keywords: fiber Bragg gratings, temperature sensors, cryogenic temperatures, pressurized fiber Bragg gratings

\section{INTRODUCTION}

Fiber Bragg gratings (FBGs) have been extensively studied and utilized for temperature and strain sensing in health monitoring systems of aerospace vehicles and other applications. ${ }^{1-3}$ Compared with other types of sensors, FBGs have the advantages of being lightweight and flexible, and also requiring simpler wiring especially for distributed sensing systems. FBGs are also candidates for multiple parameter sensing. Several techniques have been developed for simultaneous measurements of strain and temperature; however, they can be applied only to a finite range of temperatures. ${ }^{4-6}$ In general, strain response of FBGs is linear for a broad range of strain and independent of temperature. $^{7}$ The temperature response of FBGs is much more complicated. The temperature sensitivity of FBGs is moderate and relatively linear for temperatures above $273 \mathrm{~K}$. However, it becomes more nonlinear as temperature is reduced significantly below $273 \mathrm{~K}$ and much smaller for cryogenic temperatures. There are additional problems of wavelength shift continuity with FBGs in the cryogenic temperature range during a cooling process. Thus further advances in FBG sensing technology are required to enable the use of FBGs as cryogenic temperature sensors. Two key advances are needed: (1) the temperature sensitivity of the FBG sensors at cryogenic conditions must be increased; and (2) the FBG signal waveforms, which are used as indicators of temperature, must be stable and well defined.

It has been suggested that FBGs be embedded in or bonded to substrates to enhance their temperature sensitivity. ${ }^{8,9}$ These substrates, e.g., poly(methyl methacrylate) (PMMA) or Teflon, have larger thermal expansion coefficients than silica fibers. Bonding a fiber to a substrate to effect greater temperature sensitivity requires the substrate thickness to be much greater than the diameter of the fiber and the substrate length to be longer than the FBG. Therefore dimensions of these substrates are on the order of several millimeters to centimeters. While the enhancement of FBG temperature sensitivity is significant using these techniques, the flexibility of the optical fiber is sacrificed because of the need for bonding to a rigid substrate. This is particularly unsuitable for distributed sensing with multiple sensors.

*Meng-Chou.Wu-1@nasa.gov 
This study investigated the effects of polymer coatings on FBG temperature sensitivity. Coating thicknesses were varied from 11 to 62 microns but did not compromise the flexibility of the optical fibers. A physical model was developed to relate the temperature sensitivities of FBGs to coating thickness. Trends predicted by the physical model were compared to experimental results. The use of pressurized fiber Bragg gratings (PFBGs) for enhancing temperature sensitivity was also investigated. PFBGs can be obtained by applying a small diametric load to regular FBGs. For the present work, a pressure-induced transition of the PFBGs occurred at approximately $200 \mathrm{~K}$ and resulted in higher temperature sensitivity for PFBGs than FBGs at cryogenic temperatures. This intriguing phenomenon was independent of the polymer coatings and attributed to a change of the optical fiber thermo-optic coefficient. The Bragg wavelengths of PFBGs are given over the range of $4.2 \mathrm{~K}$ to $295 \mathrm{~K}$ and compared with those of regular FBGs with various coating thicknesses.

\section{THEORY}

In general a fiber Bragg grating can be characterized by its Bragg wavelength, which is the center wavelength of the light reflected from the grating. The Bragg wavelength is given as

$$
\lambda_{\mathrm{B}}=2 \mathrm{n}_{\mathrm{eff}} \Lambda,
$$

where $\mathrm{n}_{\text {eff }}$ is the effective refractive index of the fiber core and $\Lambda$ the grating period. For a fiber Bragg grating coated with polymer, a change in the temperature causes a change in the grating period due to not only the thermal expansion of the fiber but also the strain, $\delta l / l$, induced by thermal expansion of the coating polymer. In addition, the refractive index of the fiber core changes because of the thermo-optic effect. Combining all the above effects, the shift in the Bragg wavelength due to finite temperature change, $\Delta T$, is given as

$$
\delta \lambda_{\mathrm{B}} / \lambda_{\mathrm{B}}=\alpha_{\mathrm{s}} \Delta T+\left(1-\mathrm{p}_{\mathrm{e}}\right) \delta l / l+\delta \mathrm{n}_{\mathrm{eff}} / \mathrm{n}_{\mathrm{eff}},
$$

where $\alpha_{s}$ is the thermal expansion coefficient, and $p_{e}$ the photoelastic constant of the fiber, i.e., silica glass.
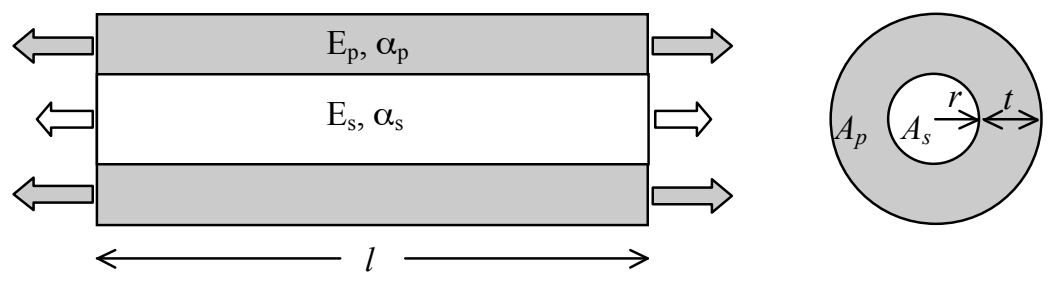

Fig. 1. Geometry defining the strain induced by thermal expansion of the fiber and the coating polymer, (a) the side view, and (b) the cross-section view. $\alpha_{\mathrm{p}}$ and $\alpha_{\mathrm{s}}$ are the thermal expansion coefficients, $\mathrm{E}_{\mathrm{p}}$ and $\mathrm{E}_{\mathrm{s}}$ the Young's moduli, and $A_{p}$ and $A_{s}$ the cross-sectional areas of the polymer and the silica glass respectively. $r$ is the radius of the fiber and $t$ is the radial thickness of the polymer respectively.

As defined by the geometry shown in the Fig.1, the strain, $\delta l / l$, induced by thermal expansion of the coating polymer can be expressed as

$$
\delta l / l=\left(\alpha_{\mathrm{p}}-\alpha_{\mathrm{s}}\right)\left[\gamma \mathrm{E}_{\mathrm{p}} /\left(\gamma \mathrm{E}_{\mathrm{p}}+\mathrm{E}_{\mathrm{s}}\right)\right] \Delta T,
$$


where $\alpha_{p}$ is the thermal expansion coefficient of the polymer, $E_{p}$ and $E_{s}$ are the Young's moduli of the polymer and silica glass respectively, and $\gamma$ is defined as

$$
\gamma \equiv A_{p} / A_{s}=\left[(r+t)^{2}-r^{2}\right] / r^{2},
$$

the ratio of the cross-sectional area, $A_{p}$, of the polymer with a radial thickness of $t$ to the cross-sectional area, $A_{s}$, of the fiber with a radius of $r$. Eq. (3) can be further reduced as

$$
\delta l / l=\left(\alpha_{\mathrm{p}}-\alpha_{\mathrm{s}}\right) \eta \Delta T
$$

where

$$
\eta \equiv \gamma \mathrm{E}_{\mathrm{p}} /\left(\gamma \mathrm{E}_{\mathrm{p}}+\mathrm{E}_{\mathrm{s}}\right)
$$

For a finite temperature change $\Delta T$, so that both the thermal expansion coefficients and the index change are linear, Eq. (2) can be written as

$$
\delta \lambda_{\mathrm{B}} / \lambda_{\mathrm{B}}=\left[\alpha_{\mathrm{s}}+\left(1-\mathrm{p}_{\mathrm{e}}\right)\left(\alpha_{\mathrm{p}}-\alpha_{\mathrm{s}}\right) \eta+\xi\right] \Delta T,
$$

where $\xi=\left(1 / \mathrm{n}_{\mathrm{eff}}\right)\left(\partial \mathrm{n}_{\mathrm{eff}} / \partial T\right)$, is the thermo-optic coefficient of the fiber.

It is of interest to note that when $t>>r, \gamma>>1$ and $\eta \approx 1$ (also, in general $\alpha_{\mathrm{p}}>\alpha_{\mathrm{s}}$ ), and therefore Eq. (7) is reduced to ${ }^{8}$

$$
\delta \lambda_{\mathrm{B}} / \lambda_{\mathrm{B}}=\left[\left(1-\mathrm{p}_{\mathrm{e}}\right) \alpha_{\mathrm{p}}+\xi\right] \Delta T,
$$

for the case of a fiber Bragg grating embedded in or bonded on a thick substrate of polymer. If $t=0, \eta=0$, then Eq. (7) becomes the well-know formula for a bare fiber grating sensor,

$$
\delta \lambda_{\mathrm{B}} / \lambda_{\mathrm{B}}=\left(\alpha_{\mathrm{s}}+\xi\right) \Delta T .
$$

In general, the second term of Eq. (7) gives the effect of the thickness of the coating on the temperature sensitivity of a fiber Bragg grating.

The third term of Eq. (7) is from the contribution of thermo-optic effect to the Bragg wavelength shift due to temperature change. Under normal conditions, it is about the same order of magnitude as the thermal expansion of the fiber from the room temperature down to the cryogenic temperatures ${ }^{7}$; however, when a diametric load is applied to the fiber, a pressure-induced transition occurs in the fiber core at a certain temperature during the cooling cycle, and therefore the thermo-optic coefficient of the fiber core has a dramatic increase. This particular effect is to be discussed in the following sections.

\section{EXPERIMENT}

A pulsed $\mathrm{KrF}$-excimer laser of $248 \mathrm{~nm}$ and a Talbot interferometer arrangement were used to write the FBGs. The interferometer consisted of a phase mask functioning as a beam splitter and a pair of mirrors used to recombine the split beams to form an interference pattern. An aperture placed in the laser beam path was used to control the grating length and spatial profile. ${ }^{10}$ FBG lengths for the present study were nominally $5 \mathrm{~mm}$. The grating pitch written into the fiber could be adjusted by changing the relative angle of the two mirrors. This optical setup was mounted on the NASA Langley optical fiber draw tower to allow FBGs to be written into the optical fiber as the fiber was being drawn. The single-mode fibers used in this study were drawn from commercially available germanium-doped preforms of high numerical apertures. The drawn fibers with FBGs were coated with polyimide to thicknesses ranging from 11 to 16 micrometers, then ink-marked to show grating locations. Larger coating thicknesses were obtained for the 
purposes of this study by recoating the drawn fiber with thermally cured polyimide or UV-cured acrylic. The UVcured acrylic was used in cases where a thicker coating of polyamide would be difficult to apply.

These low reflectivity FBGs (single grating reflectivity smaller than a few tenths of one percent) were interrogated using a frequency domain demodulation system ${ }^{11}$ shown in Fig. 2. In this system, the fiber coupler $\mathrm{C}_{1}$ and a pair of Faraday rotation mirrors (FRMs) form an in-fiber interferometer with an optical path difference of $2 \mathrm{n}_{\text {eff }} \mathrm{L}_{0}$, where $\mathrm{n}_{\text {eff }}$ is the effective refractive index of the fiber core and $\mathrm{L}_{0}$ the length of the reference cavity. The signals are driven by the tuning of the laser and detected at the photo-detector $\mathrm{D}_{1}$. They are used to trigger the sampling of signal at $\mathrm{D}_{2}$, which is the output of another in-fiber interferometer formed with the fiber coupler $\mathrm{C}_{2}$, a broadband reflector, and a particular fiber Bragg grating at a distance of $\mathrm{L}_{\mathrm{i}}$. If there are a series of low reflectivity Bragg gratings written at the same wavelength on a single fiber at different locations, the reflected signals from each grating are superimposed and detected at $\mathrm{D}_{2}$. The detected signals are further processed (fast-Fourier-transformed) to obtain the spatial spectrum of all gratings, which displays the physical profiles of the gratings at different locations. The spatial spectrum of a particular grating can then be windowed and inverse-fast-Fourier-transformed to get its own wavelength spectrum. Fig. 3(a) shows the spatial spectrum of two FBGs in a fiber with multiple gratings. The gratings have a physical length of about $5 \mathrm{~mm}$ and a separation distance of $10 \mathrm{~cm}$. Two lines in Fig. 3(a) indicate the window of the inverse FFT for the second FBG. The calculated wavelength spectrum of the second FBG gives a center wavelength of $1551.3 \mathrm{~nm}$, as shown in Fig. 3(b).

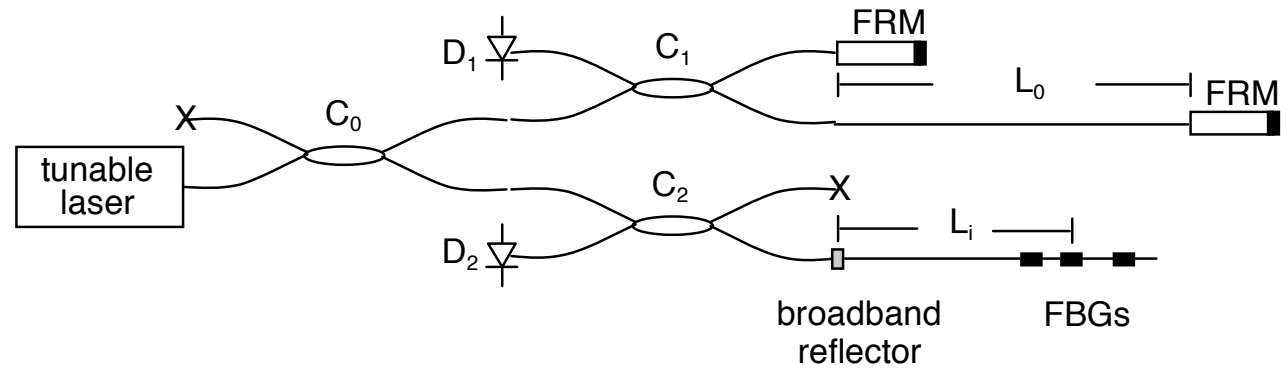

Fig. 2. Schematic diagram of a frequency domain demodulation system. Items $C_{0}, C_{1}$, and $C_{2}$ are fiber couplers. "X" indicates that the unused port is terminated. Items $\mathrm{D}_{1}$ and $\mathrm{D}_{2}$ are detectors and FRMs are Faraday rotation mirrors.
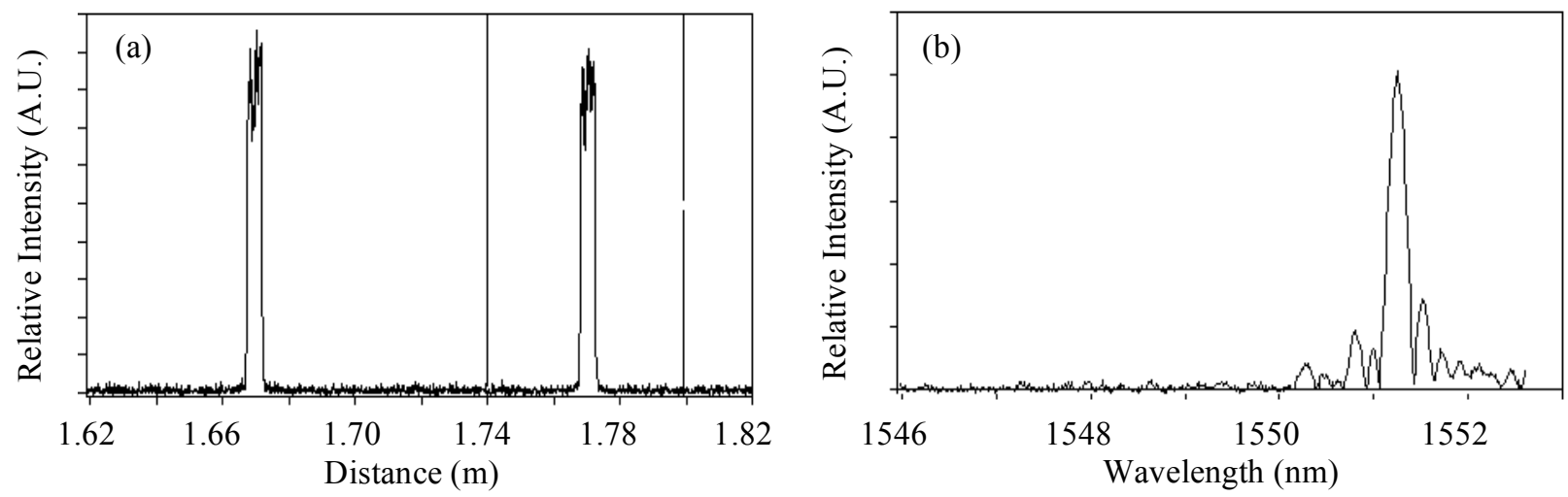

Fig. 3. (a) The spatial spectrum of two FBGs with a physical length of about $5 \mathrm{~mm}$ and two lines indicating the window of the inverse FFT for the second FBG. (b) The calculated wavelength spectrum of the second FBG with a center wavelength of $1551.3 \mathrm{~nm}$. 
A cryostat of compressed helium gas was used for temperature control, ranging from $350 \mathrm{~K}$ to about $8 \mathrm{~K}$. The optical fiber with the investigated Bragg grating was held between the cold plate of the cryogenic chamber and another copper plate with a length of about $2 \mathrm{~cm}$ to cover the whole Bragg grating. As shown in the Fig. 4, four screws with four identical springs were used to hold the plates and apply a small diametric load to the fiber to form a pressurized Bragg grating (PFBG). A section of support fiber of equal diameter with the test fiber was added to keep the two plates parallel. The total force applied by the springs to the copper plate was approximately $15 \mathrm{~N}$, calculated from the spring constant at room temperature. For the whole range of the temperature change, the total variations of the spring constant and the dimension changes of the copper plates and the steel screws were estimated to be less than a few percents. The applied force was sufficient to reach the threshold stress required for a PFBG but was kept less than 30 $\mathrm{N}$ to prevent splitting of the center peak in the wavelength spectrum. ${ }^{12}$ Data were acquired periodically throughout the cooling cycle using the frequency domain demodulation system described previously. The time required to cool from room temperature to the lowest temperature of $8 \mathrm{~K}$ was approximately two hours.

For comparison, fiber Bragg gratings with and without coating were also immersed directly in liquid nitrogen and liquid helium to measure their Bragg wavelength shifts.

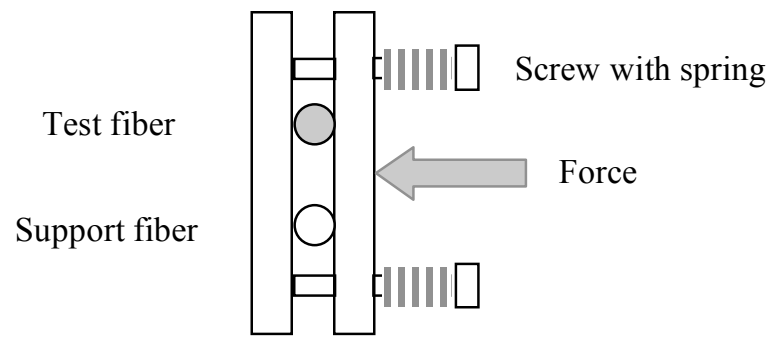

Fig. 4. Schematic representation of a pressurized fiber Bragg grating (PFBG). A copper plate with four screws holds the test fiber and the support fiber onto the cold plate of the cryogenic chamber. Four springs apply a diametric load to the test fiber to form a PFBG.

\section{RESULTS AND DISCUSSION}

Fig. 5 shows the measured Bragg wavelength versus temperature for a regular FBG with polyimide coating, which has a thickness of about 11 micrometers. The FBG was only attached to the cold plate for temperature control without being pressurized. For temperatures from $295 \mathrm{~K}$ to $200 \mathrm{~K}$, the temperature sensitivity of the regular FBG is about 8 $\mathrm{pm} / \mathrm{K}$; for temperatures ranging from $200 \mathrm{~K}$ to $80 \mathrm{~K}$ the coefficient is more nonlinear and becomes smaller as the temperature decreases; and for temperatures below $80 \mathrm{~K}$ the curve is almost flat, i.e., showing zero temperature sensitivity. There are also some problems of wavelength shift continuity with FBGs for the cryogenic measurements. During the cooling process, the center peak of an FBG might shift as usual but then suddenly move backwards, as shown in Fig. 5 around $90 \mathrm{~K}$. This change in wavelength shift would cause an invalid measurement. Therefore, it seems to be impractical, if not totally impossible, to use ordinary FBGs with thin coatings as cryogenic temperature sensors.

Also shown in Fig. 5 is the measured Bragg wavelength as a function of temperature for the PFBG with $11 \mu \mathrm{m}$ polyimide coating - the same thickness as the FBG. The temperature sensitivity of the PFBG for temperatures from $295 \mathrm{~K}$ to $200 \mathrm{~K}$ is about $9 \mathrm{pm} / \mathrm{K}$, not significantly greater than that of the regular FBG. However, at about $200 \mathrm{~K}$, a pressure-induced transition occurs. Below $200 \mathrm{~K}$ the temperature coefficient of the PFBG reaches $24 \mathrm{pm} / \mathrm{K}$, more than three times the regular FBG. Moreover, for the temperature change from $80 \mathrm{~K}$ to $10 \mathrm{~K}$, the PFBG has a total wavelength shift of about $470 \mathrm{pm}, 10$ times more than the regular FBG. 


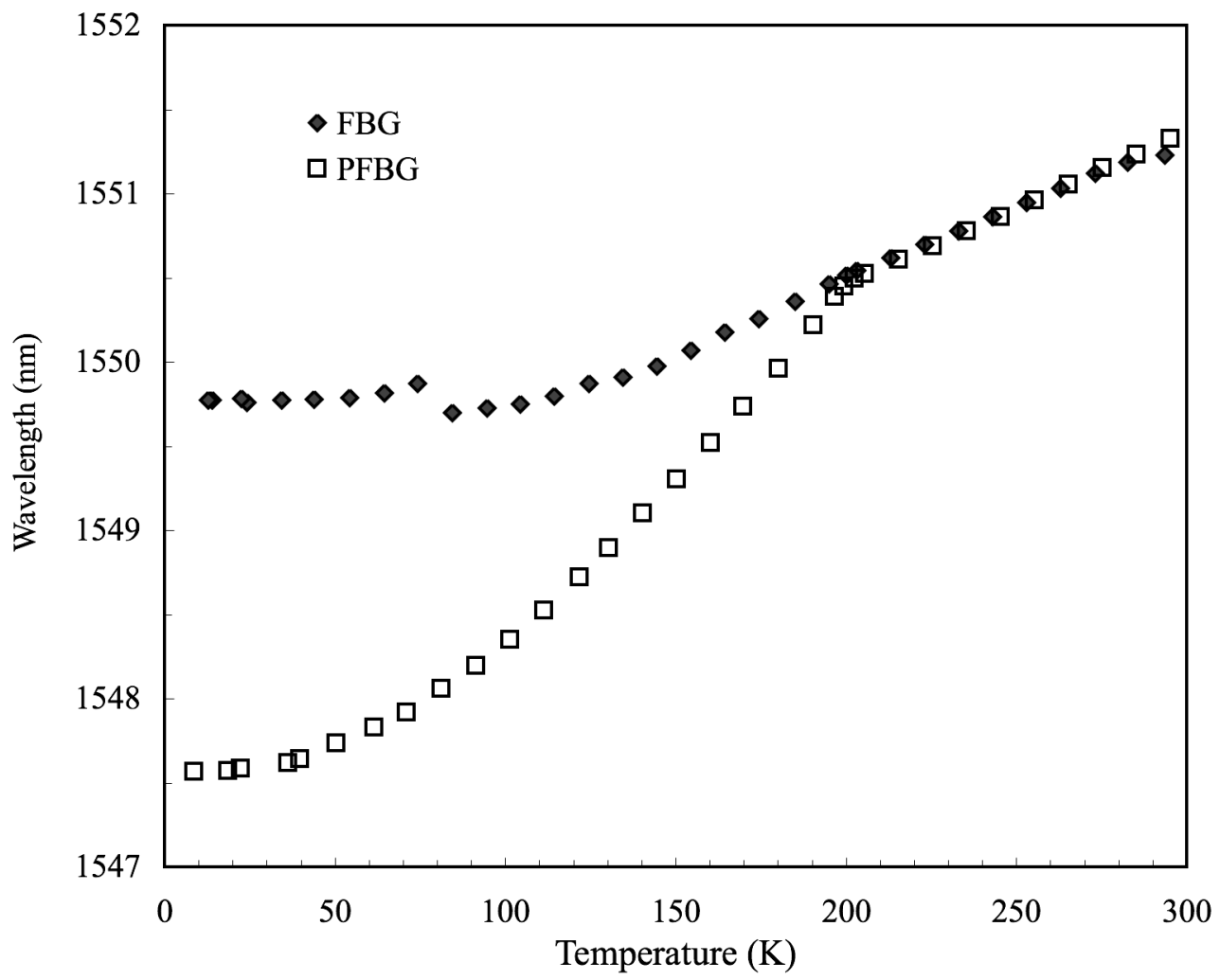

Fig. 5. Bragg wavelength shifts of a regular FBG and a PFBG with temperature during a cooling cycle. The coating thickness is $11 \mu \mathrm{m}$ for each.

Fig. 6 shows the Bragg wavelength shifts of the same PFBG (11 $\mu \mathrm{m}$ coating) during the cooling and the heating cycles. The heating cycle has a heating rate of about $4 \mathrm{~K} / \mathrm{min}$ for the temperature increasing from $10 \mathrm{~K}$ to $295 \mathrm{~K}$. At cryogenic temperatures the PFBG has the similar thermal response of heating as that of cooling, except only a very small amount of hysteresis exists. However, during the heating cycle, as the temperature passes $200 \mathrm{~K}$, the transition temperature, the Bragg wavelength keeps increasing at the same rate until the temperature reaches about $210 \mathrm{~K}$, then decreases around $220 \mathrm{~K}$ and increases again at about $230 \mathrm{~K}$. This overshooting indicates the relaxation time of the pressure-induced transition might be longer than the time scale.

Table 1 shows the Bragg wavelength shifts of the FBGs with various coating thicknesses as they were immersed in liquid nitrogen and liquid helium directly from room temperature. The FBG without coating exhibited a Bragg wavelength shift of $\Delta \lambda_{\mathrm{B}}=1.36 \mathrm{~nm}$ when immersed in liquid nitrogen at $77 \mathrm{~K}$ and $\Delta \lambda_{\mathrm{B}}=1.37 \mathrm{~nm}$ when immersed in liquid helium at $4.2 \mathrm{~K}$. The FBG with $11 \mu \mathrm{m}$ polyimide coating gives $\Delta \lambda_{\mathrm{B}}=1.47 \mathrm{~nm}$ when immersed in liquid nitrogen and $\Delta \lambda_{B}=1.51 \mathrm{~nm}$ for liquid helium. These values of $\Delta \lambda_{B}$ observed for direct immersion of the $11 \mu \mathrm{m}-$ coated FBG in liquid nitrogen and liquid helium are consistent with the value of $\Delta \lambda_{B} \approx 1.51$ observed by cooling an identical FBG with $11 \mu \mathrm{m}$ coating from room temperature to $\sim 8 \mathrm{~K}$ using the cryostat, as indicated by the data in Fig. 5 . The difference between the results with and without coating is apparently attributed to the thermal expansion of the polymer. Furthermore, as described in the physical model of the section 2, Eq. (7), Table 1 shows the Bragg wavelength shifts increase with increasing coating thickness. For thicker coating the increase is more significant, although the wavelength shifts between the liquid nitrogen and liquid helium temperatures are still minimal. This indicates the temperature sensitivity of a FBG with thicker coating might be adequate for cryogenic temperature sensing down to the $77 \mathrm{~K}$ temperature of liquid nitrogen. However, even with the thicker coating, the FBG thermal sensitivity may not be sufficient for temperature sensing for temperatures below $77 \mathrm{~K}$. 


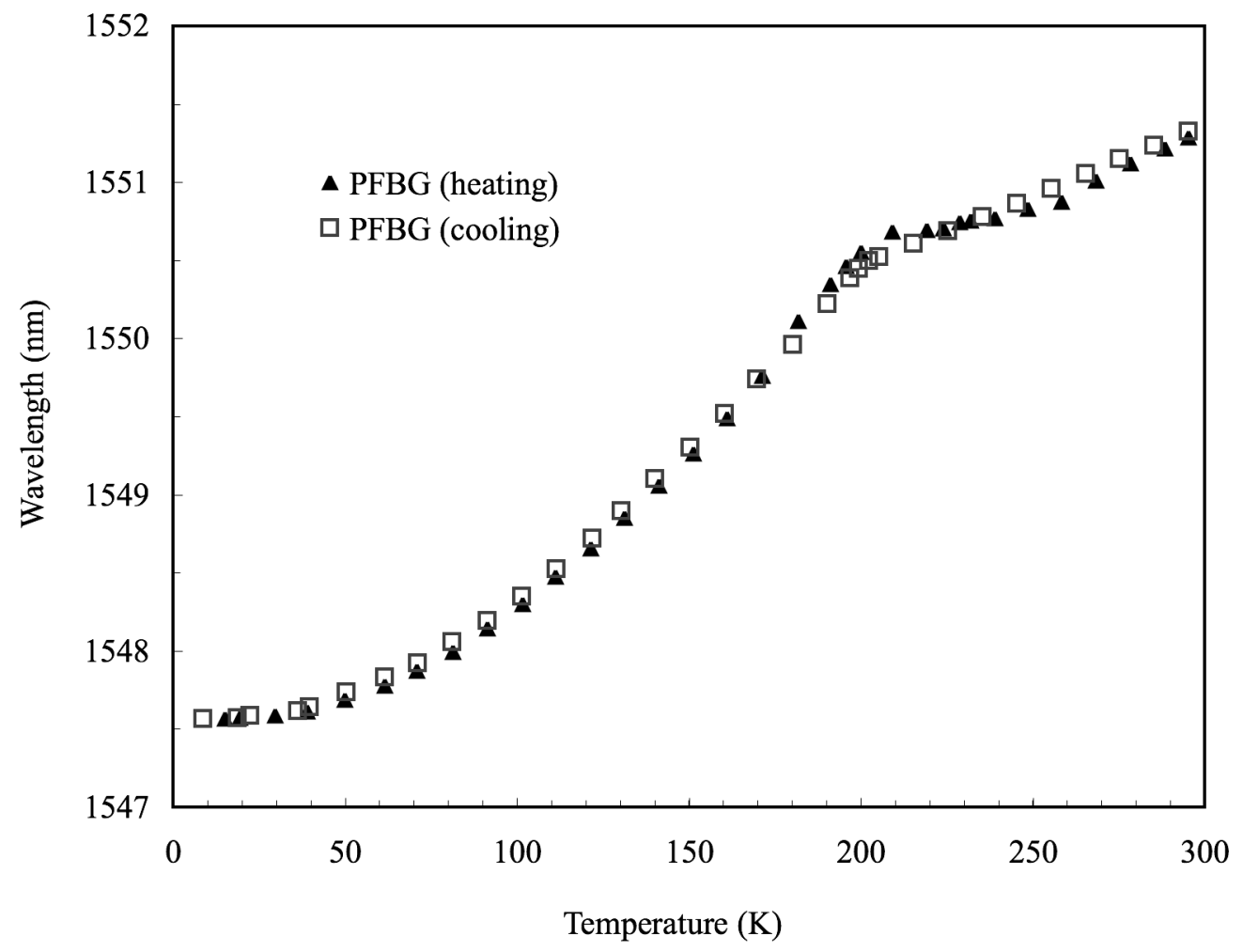

Fig. 6. Bragg wavelength shifts of a PFBG (11 um coating) with temperature during cooling and heating cycles.

Table 1. The Bragg wavelength shifts of FBGs immersed in liquid nitrogen and liquid helium

\begin{tabular}{|c|c|c|}
\hline \multirow{2}{*}{$\begin{array}{c}\text { FBGs } \\
\text { (with coating thickness, } t \text { ) }\end{array}$} & \multicolumn{2}{|c|}{$\begin{array}{c}\Delta \lambda_{\mathrm{B}}(\mathrm{nm}) \text { from room temperature } \\
\text { to }\end{array}$} \\
\hline & Liquid-N ${ }_{2}(77 \mathrm{~K})$ & Liquid-He $(4.2 \mathrm{~K})$ \\
\hline $\mathrm{FBG}(\mathrm{t}=0)$ & 1.36 & 1.37 \\
\hline $\operatorname{FBG}(\mathrm{t}=11 \mu \mathrm{m})^{\mathrm{a}}$ & 1.47 & 1.51 \\
\hline $\operatorname{FBG}(\mathrm{t}=22 \mu \mathrm{m})^{\mathrm{a}}$ & 1.77 & 1.99 \\
\hline $\operatorname{FBG}(\mathrm{t}=62 \mu \mathrm{m})^{\mathrm{b}}$ & 3.09 & 3.38 \\
\hline
\end{tabular}

Table 2 shows the total Bragg wavelength shifts of FBGs and PFBGs with various coating thicknesses from room temperature to about $8 \mathrm{~K}$. The wavelength shifts of PFBGs are greater than those of FBGs and all increase with increasing coating thickness. The differences of wavelength shifts between PFBGs and FBGs $\left(\Delta \lambda_{B}{ }^{\text {PFBG }}-\Delta \lambda_{B}{ }^{F B G}\right)$ for each thickness are shown in the third column. These differences in Bragg wavelength shift are consistently near 2.2 $\mathrm{nm}$, independent of the coating thickness. This extra wavelength shift resulting from the pressure-induced transition is apparently attributed to the change of the thermo-optic properties of the fiber core, i.e., the change of the refractive index, which is related to the energy level of germanium defects. ${ }^{13}$ These effects are similar to those affecting the energy levels of semiconductors, ${ }^{14}$ although details of the mechanisms for the transition need further investigation. 
Table 2. Comparison of the total Bragg wavelength shifts of FBGs and of PBFGs from room temperature to $8 \mathrm{~K}$ for various coating thicknesses

\begin{tabular}{|c|c|c|c|}
\hline Coating thickness, $\mathrm{t}$ & $\Delta \lambda_{\mathrm{B}}{ }^{\mathrm{FBG}}(\mathrm{nm})$ & $\Delta \lambda_{\mathrm{B}}{ }^{\text {PFBG }}(\mathrm{nm})$ & $\Delta \lambda_{\mathrm{B}}{ }^{\text {PFBG }}-\Delta \lambda_{\mathrm{B}}{ }^{\mathrm{FBG}}$ \\
\hline $11 \mu \mathrm{m}$ & 1.51 & 3.78 & 2.27 \\
\hline $22 \mu \mathrm{m}$ & 1.99 & 4.15 & 2.16 \\
\hline $62 \mu \mathrm{m}^{\mathrm{a}}$ & 3.38 & 5.60 & 2.22 \\
\hline
\end{tabular}

${ }^{\mathrm{a}}$ Same as in the Table 1 .

\section{CONCLUSION}

We have developed a physical model to relate the Bragg wavelength shifts of FBGs to the thermal expansion coefficients, Young's moduli, and radial thicknesses of the coating polymers. The experimental results show the temperature sensitivities of FBGs increase with increasing coating thickness. This relationship might further enable the coating materials to have sensing capabilities when combined with FBGs.

We have also demonstrated a technique of using pressurized FBGs for high sensitivity temperature sensors. PFBGs can be obtained by applying a small diametric load to regular FBGs. Compared to a regular FBG, a PFBG has slightly greater temperature sensitivity for the temperatures from $295 \mathrm{~K}$ to $200 \mathrm{~K}$. At $200 \mathrm{~K}$, a pressure-induced transition occurs and the temperature sensitivity of the PFBG below the transition temperature reaches three times as large as that obtained with the FBG. For temperatures below $80 \mathrm{~K}$, the PFBG, although showing nonlinearity, has 10 times more wavelength shift than the regular FBG. The increases of the wavelength shifts from FBGs to PFBGs are independent of the coatings thickness and are attributed to the changes of the fiber thermo-optic coefficients.

FBGs can be coated with polymer to a thickness that enhances the temperature sensitivity and also retains the flexibility of optical fibers. The purpose of using the copper plates to form a PFBG in this study was to provide the threshold stress required for the grating to complete the transition. Since the required load is small, it can be obtained with a simple clamping device or cladding on fibers. The enhanced temperature sensitivity of PFBGs makes them suitable for distributed cryogenic temperature sensing.

\section{ACKNOWLEDGEMENTS}

The authors thank Dr. Russell A. Wincheski and late Dr. Keun J. Sun for their assistance of using liquid helium for part of immersion measurements.

\section{REFERENCES}

1. C. R. Giles, "Lightwave applications of fiber Bragg gratings," J. Lightwave Technol. 15, 1391-1404 (1997)

2. K. O. Hill, Y. Fujii, D. C. Johnson, and B. S. Kawasaki, "Photosensitivity in optical waveguides: application to reflection filter fabrication, “ Appl. Phys. Lett. 32, 647-649 (1978)

3. A. D. Kersey, M. A Davis, H. J. Patrick, M. LeBlanc, K. P. Koo, C. G. Askins, M.A. Putnam, E. J. Friebele, "Fiber grating sensors," J. Lightwave Technol. 15, 1442-1463 (1997)

4. M. G. Xu, J.-L. Archambault, L. Reekie, and J. P. Dakin, "Discrimination between strain and temperature effects using dual-wavelength fiber grating sensors," Electron. Lett. 31, 823-825 (1994).

5. S. W. James, M. L. Dockney, and R. P. Tatam, "Simultaneous independent temperature and strain measurement using in-fiber Bragg grating sensors," Electron. Lett. 32, 1133-1134 (1996).

6. H. J. Patrick, G. M. Williams, A. D. Kersey, and J. R. Pedrazzani, "Hybrid fiber Bragg grating/long period fiber grating sensor for strain/temperature discrimination,” IEEE Photon. Technol. Lett. 8, 1223-1225 (1996). 
7. S. W. James, R. P. Tatam, A. Twin, M. Morgan, and P. Noonan, "Strain response of fiber Bragg grating sensors at cryogenic temperatures," Meas. Sci. Technol. 13, 1535-1539 (2002)

8. S. Gupta, T. Mizunami, T. Yamao, and T. Shimomura, "Fiber Bragg grating cryogenic temperature sensors," Appl. Opt. 25, 5202-5205 (1996)

9. T. Mizunami, H. Tatehata, and H. Kawashima, "High-sensitivity cryogenic fiber-Bragg-grating temperature sensors using Teflon substrates,” Meas. Sci. Technol. 12, 914-917 (2001)

10. M.-C. Wu and R. S. Rogowski, "Fabrication of self-apodized short-length fiber Bragg gratings," Appl. Opt. 42, 5017-5023 (2003)

11. M. Froggatt and J. Moore, "Distributed measurement of static strain in an optical fiber with multiple Bragg gratings at nominally equal wavelengths," Appl. Opt. 37, 1741-1746 (1998)

12. R. B. Wagreich, W. A. Atia, H. Singh, and J. S. Sirkis, "Effects of diametric load on fiber Bragg gratings fabricated in low birefringent fiber," Electron. Lett. 32, 1223-1224 (1996)

13. A. Othonos and K. Kalli, Fiber Bragg Gratings: Fundamentals and Applications in Telecommunications and Sensing, Artech House, Boston, 1999.

14. J. I. Pankove, Optical Processes in Semiconductors, Dover Publications, New York, 1975.

\section{APPENDIX A. DERIVATION OF EQ. (3), THE THERMAL STRAIN OF A POLYMER-COATED FIBER}

For a polymer-coated fiber, when the temperature changes, the total strain of the polymer, $\delta l_{p} / l$, and of the silica glass, $\delta l_{s} / l$, can be written respectively as

$$
\delta l_{p} / l=\alpha_{\mathrm{p}} \Delta T+\tau_{\mathrm{p}} / \mathrm{E}_{\mathrm{p}}
$$

and

$$
\delta l_{s} / l=\alpha_{\mathrm{s}} \Delta T+\tau_{\mathrm{s}} / \mathrm{E}_{\mathrm{s}}
$$

where $\alpha_{p}$ and $\alpha_{s}$ are the thermal expansion coefficients, $E_{p}$ and $E_{s}$ are the Young's moduli, and $\tau_{p}$ and $\tau_{s}$ are the induced stresses on the cross-sectional areas of the polymer and silica glass respectively. The first term of Eq. (A.1) or Eq. (A.2) is the thermal expansion of the material and the second term is the thermal strain induced by the other material. If there is no disbonding of the polymer form the silica fiber when the temperature changes, or based on the condition of continuity, both the strains should be the same, i.e., $\delta l_{\mathrm{p}} / l=\delta l_{s} / l$, or

$$
\alpha_{\mathrm{p}} \Delta T+\tau_{\mathrm{p}} / \mathrm{E}_{\mathrm{p}}=\alpha_{\mathrm{s}} \Delta T+\tau_{\mathrm{s}} / \mathrm{E}_{\mathrm{s}} .
$$

Eq. (A.3) can be rewritten as

$$
\left(\alpha_{p}-\alpha_{s}\right) \Delta T+\left(\tau_{p} / E_{p}-\tau_{s} / E_{s}\right)=0 .
$$

Since no external forces exist,

$$
\tau_{\mathrm{p}} A_{p}+\tau_{\mathrm{s}} A_{s}=0,
$$

or

$$
\tau_{\mathrm{s}}=-\left(A_{p} / A_{s}\right) \tau_{\mathrm{p}} \equiv-\gamma \tau_{\mathrm{p}},
$$

where $\gamma$ is the ratio of the cross-sectional area, $A_{p}$, of the polymer with a radial thickness of $t$ to the cross-sectional area, $A_{s}$, of the fiber with a radius of $r$ as defined in Eq. (4). $A_{p}$ and $A_{s}$ also change with the changes of temperature. However, for a finite temperature change, these second order effects are negligible when calculating $\gamma$, i.e., $\gamma(T) \approx \gamma$. 
By using Eq. (A.6), Eq. (A.4) can be rewritten as

$$
\left(\alpha_{p}-\alpha_{s}\right) \Delta T-\tau_{s}\left[1 /\left(\gamma E_{p}\right)+1 / E_{s}\right]=0,
$$

then

$$
\tau_{\mathrm{s}}=\left(\alpha_{\mathrm{p}}-\alpha_{\mathrm{s}}\right) \Delta T\left[\left(\gamma \mathrm{E}_{\mathrm{p}} \mathrm{E}_{\mathrm{s}}\right) /\left(\gamma \mathrm{E}_{\mathrm{p}}+\mathrm{E}_{\mathrm{s}}\right)\right] .
$$

Therefore, the thermal strain on the silica glass induced by the polymer is given by

$$
\tau_{\mathrm{s}} / \mathrm{E}_{\mathrm{s}} \equiv \delta l / l=\left(\alpha_{\mathrm{p}}-\alpha_{\mathrm{s}}\right)\left[\gamma \mathrm{E}_{\mathrm{p}} /\left(\gamma \mathrm{E}_{\mathrm{p}}+\mathrm{E}_{\mathrm{s}}\right)\right] \Delta T,
$$

which is equivalent to Eq. (3). 